\title{
Determinantes, Procesos y Resultados de la Formación Inicial de Profesores de Enseñanza Básica y Educación Parvularia en Chile: Una Aproximación Conceptual
}

\author{
Emilio R. Rodríguez-Ponce ${ }^{(1) \star}$, Liliana M. Pedraja-Rejas ${ }^{(2)}$ y Francisco A. Ganga-Contreras ${ }^{(3)}$ \\ (1) Instituto de Alta Investigación, Universidad de Tarapacá. Casilla 7-D, Arica-Chile. (e-mail: erodriguez@uta.cl) \\ (2) Departamento de Ingeniería Industrial y de Sistemas, Universidad de Tarapacá, Casilla 7-D, Arica-Chile. \\ (e-mail: Ipedraja@uta.cl) \\ (3) Departamento de Ciencias del Desarrollo, Universidad de Los Lagos, Chile. (e-mail: fganga@ulagos.cl)
}

* Autor a quien debe ser dirigida la correspondencia

Recibido Dic. 27, 2018; Aceptado Mar. 15, 2019; Versión final Jun. 18, 2019, Publicado Dic. 2019

\begin{abstract}
Resumen
Se identifica mediante una aproximación teórica, qué factores son determinantes para explicar los resultados académicos de los procesos de formación inicial docente de Profesores de Enseñanza Básica y Educación Parvularia en Chile. Para este fin se postula un conjunto de hipótesis las que permiten construir mediante inducción analítica un modelo integrador en el que los resultados académicos de ambas carreras estarían determinados tanto por las condiciones de entrada de los estudiantes a la universidad como por los procesos académicos. Así, se propone desde el punto de vista conceptual que diferentes relaciones entre condiciones de entrada y procesos académicos conducirán a resultados académicos. Se concluye que la originalidad del artículo viene dada por la propuesta del modelo que emerge del análisis conceptual profundo basado en la literatura del campo de la educación.
\end{abstract}

\section{Determinants, Processes and Results of the Teacher Education of Early Childhood Teachers and Primary Teachers in Chile: A Conceptual Approach}

\begin{abstract}
Through a theoretical approach, the relationships between determinants, processes and results of the teachers of elementary and early childhood education are identified. A set of hypotheses is proposed, which, in turn, allow the construction of an integrative model through analytical induction. This theoretical research consists of proposing that the academic results of both careers would be determined both by the conditions of entrance of the students to the university and by the academic processes. Thus, it is postulated that different relationships between entry conditions and academic processes will lead to differentiated academic results, each other, systemically. It is concluded that the originality of the article is given by the proposal of the model that emerges from the conceptual analysis based on the literature of the field of education.
\end{abstract}

Keywords: teacher education; teachers; conceptual approach; early childhood education; elementary education; academic results 


\section{INTRODUCCIÓN}

La fuente de la ventaja competitiva de los países para insertarse en la sociedad globalizada del conocimiento y la información radica principalmente en la formación de capital humano avanzado y en las capacidades que se desarrollan para la creación, difusión y aplicación del conocimiento. De allí que los procesos educativos en los primeros años de vida sean determinantes de las opciones de progreso y desarrollo de las personas y de su potencial para contribuir, en el futuro, al desarrollo del país (Magnuson y Duncan, 2016). Así, la Educación Parvularia tiene un rol esencial, ya que tal como lo plantea el Council for Early Childhood Development (2011) en los primeros cuatro años de vida la sensibilidad del cerebro para aprender números, lenguaje, símbolos, habilidades sociales y control emocional llega a su máximo potencial; mientras que, en los años posteriores, dicha sensibilidad disminuye de manera significativa. En la misma línea, el Center on the Developing ChildHarvard University (2007), ha descubierto que un niño de nivel socio económico alto utiliza cerca de 1.200 palabras a los tres años, mientras que un niño de un nivel socioeconómico bajo no llega a dominar 400 palabras a esa misma edad. A su turno, la Educación Básica también tiene gran importancia por cuanto estudiantes promedio (percentil 50) a los ocho años de edad llegan a ser alumnos sobresalientes (percentil 90) a los once años, siempre y cuando sean formados por un profesor de alto desempeño; en cambio, estudiantes promedio a los ocho años se transforman en alumnos deficientes a los once años (percentil 37), si son formados por un profesor de bajo desempeño (Barber y Mourshed, 2007).

Las capacidades existentes en Chile para insertarse efectivamente en la sociedad del conocimiento se encuentran con una limitación crucial, dada por el bajo nivel de eficacia funcional de la población nacional. En efecto, el Centro de Microdatos de la Universidad de Chile (2014) revela que la población adulta tiene niveles altos de analfabetismo funcional en: la comprensión de textos (44\%), el entendimiento de documentos (42\%) y en el área cuantitativa (51\%) y se descubre, además, que en los últimos 15 años prácticamente no ha existido progreso alguno en estas dimensiones. Asimismo, el Programa para la Evaluación Internacional de las Competencias de Adultos (2016) reconoce que cerca de la mitad de los adultos en Chile sólo puede completar tareas básicas como la lectura de textos breves y simples, a la vez que es capaz de efectuar sólo cálculos matemáticos elementales. Por consiguiente, no resulta sorprendente que la productividad de la economía chilena ha decrecido en los últimos 5 años (OCDE, 2018).

La evidencia disponible da cuenta que las carreras, los egresados recientes y los titulados en ejercicio, en general, poseen niveles de calidad inferiores a los requeridos por una sociedad que, a su vez, exige mayor competitividad, una preparación intelectual más elevada y niveles superiores de eficacia social y funcional (Popescu et al., 2016).

Por lo tanto, se sigue que sin una educación de calidad desde la primera infancia simplemente no existirá movilidad social, ya que, en este marco, el factor determinante del futuro de las personas sería el nivel socioeconómico de la familia. En consecuencia, la Educación Parvularia y la Educación Básica constituyen la piedra angular de la preparación intelectual de las personas, así como de su posicionamiento y potencial contribución a la sociedad (Desimone y Garet, 2016). Frente a esta realidad, la Ley de Sistema de Desarrollo Profesional Docente, aprobada en el año 2016, se crea teniendo como foco central la formación inicial docente, implementación de un sistema de inducción para los docentes y una nueva carrera profesional docente.

A su vez, la ley establece que las universidades deben aplicar dos evaluaciones diagnósticas a sus estudiantes de pedagogía y educación. Una primera evaluación al inicio de la carrera: la cual debe ser aplicada por las propias universidades con el objetivo de obtener información que permita establecer mecanismos de acompañamiento y nivelación para los estudiantes, de ser necesario y una segunda evaluación Nacional Diagnóstica de la FID la cual será aplicada por el Ministerio de Educación un año antes del egreso, con el objetivo de generar información sobre los conocimientos pedagógicos, disciplinarios y didácticos obtenidos por los estudiantes durante su formación universitaria (CPEIP, 2018a).

En Chile, el último análisis realizado por la CNA respecto de las fortalezas y debilidades de las carreras de pedagogía del año 2018 indica que a inicios de junio de 2018 se cuenta con un total de 509 carreras de pedagogía impartidas en instituciones de educación superior autónomas, de las cuales solo un $64 \%$ se encontraban acreditadas (326 carreras), un 19\% no acreditadas (96 carreras) y un 17\% no se había sometido a procesos de acreditación en el sistema (87 carreras) (CNA, 2018).

Si se realiza un análisis por tipo de institución que imparte las carreras, las universidades pertenecientes al $\mathrm{CRUCH}$ tienen el mejor registro de calidad: en promedio, el $74 \%$ de su oferta se encuentra acreditada, un $10 \%$ no acreditada y un $16 \%$ fuera de sistema. En contraste, las universidades privadas poseen un $54 \%$ de su oferta de pedagogía acreditada, un $27 \%$ con decisión de no acreditación y un $19 \%$ fuera de sistema. El grueso de la oferta ( 179 carreras, $35 \%$ del total) se encuentra acreditada en el segundo tramo de acreditación 
(4 y 5 años); en el primer tramo (2 y 3 años) se encuentra el 16\% de la oferta (79 carreras); y en el tercer tramo de acreditación (6 y 7 años) se contabilizan 68 carreras (13\% de la oferta).

En efecto, el 59\% de las carreras de pedagogía forman a profesores para la educación media, un 18\% para profesores de Educación Básica, un 12\% de Educación Parvularia, y un 11\% para profesores de Educación Diferencial. Ciertamente entre los niveles hay diferencias en cuanto a sus registros de calidad: el $69 \%$ de las carreras de educación media se encuentran acreditadas; las carreras de educación parvularia y básica tiene niveles cercanos a la media ( $63 \%$ y $62 \%$ de sus carreras acreditadas); y las carreras de educación diferencial presentan la proporción más baja dentro del sistema de carreras acreditadas (46\%). Dentro de este último nivel, un tercio de las carreras nunca se ha sometido a procesos de acreditación (categoría Fuera de sistema) (CNA, 2018).

En cuanto al plan de estudios y competencias, se señala como aspecto desfavorable observado en las resoluciones de acreditación, la escasa o nula vinculación de los planes-orientado a objetivos-con el modelo por competencias declarado en el perfil de egreso de las carreras de pedagogía. Por esta razón, se justifica la necesidad de estudiar teóricamente qué factores son determinantes para explicar los resultados académicos de los procesos de formación inicial docente de Profesores de Enseñanza Básica y Educación Parvularia. Surge así la pregunta: ¿cómo se relacionan desde la perspectiva teórica los resultados académicos de las carreras en estudio con las condiciones de entrada y los procesos de formación?

Ahora bien, a partir de la generación de un enfoque integrador, que emerge de la aplicación metodológica de una revisión conceptual, debería contribuir al mejoramiento efectivo de los procesos de formación de los futuros educadores, en la perspectiva de alinear sus resultados con los objetivos de desarrollo del país. De esta forma, se resalta la posibilidad de que una contribución que ha de ser relevante para la literatura sirva también en el diseño de políticas públicas.

En definitiva, los hallazgos, a nivel teórico, de las condiciones de entrada, los procesos formativos, y los resultados permitirán aportar al acervo de conocimientos mediante la propuesta de un modelo construido a partir de inducción analítica para las dos carreras en estudio.

\section{ANTECEDENTES CONCEPTUALES}

En el campo de la educación superior, siguiendo a Vlãsceanu et al. (2004) se puede plantear que "la calidad en Educación Superior, es un concepto multidimensional, de múltiples niveles, dinámico, que se relaciona con los elementos contextuales de un modelo educacional, con la misión y fines institucionales, y con estándares específicos dentro de un sistema, institución, programa o disciplina determinados". Bajo esta perspectiva, la presente sección analiza las condiciones de entrada, los procesos de formación y los resultados académicos, que configuran sistémicamente la calidad de las carreras de Educación Parvularia y Pedagogía en Educación Básica.

\section{Las condiciones de entrada}

\section{Calidad del profesorado}

El contexto inédito de cambios que se abren a propósito de la ley de Carrera Docente es una oportunidad invaluable para afrontar este desafío: el rol de los mentores puede resultar clave para promover instancias de modelamiento y ejercitación de la práctica reflexiva; también el uso que puede darse a las horas no lectivas para constituir genuinas comunidades de aprendizaje, en que las instancias de reflexión conjunta y guiada en las escuelas se encuentren efectivamente calendarizadas y debidamente resguardadas dentro de las tareas docentes (Rodríguez et al., 2016).

La calidad de los profesores se asocia con: poseer conocimientos consistentes con una preparación académica superior; exhibir habilidades interpersonales; demostrar un compromiso con los valores sociales; y poseer capacidad de adaptación e innovación (Vasconcellos y Sordi, 2016). En concreto, la calidad del profesorado es un factor clave que impacta en el proceso de enseñanza y aprendizaje (Clark et al., 2016) y en los resultados académicos. En el ámbito de la Educación Parvularia, las investigaciones han destacado que el profesorado debe ser de la más alta calidad, por cuanto su preparación e idoneidad influirá en el desarrollo de las capacidades y conocimientos de los futuros educadores de niños de temprana edad (Garrity et al., 2016).

De hecho, la calidad del profesorado se puede asociar no sólo con los resultados de académicos, sino que también con la motivación y compromiso de quienes estudian para ser profesores (Parker et., al, 2016). Complementariamente, se sabe que el mejoramiento de la formación inicial docente requiere de regulaciones 
en la cantidad de programas que son viables y necesarios para los requerimientos docentes de Chile (Ávalos, 2014).

Sin embargo, en un contexto de masificación de la educación superior, surge el desafío de contar con la cantidad y la calidad de profesorado idóneo para acoger, interactuar e integrar a jóvenes más vulnerables y con menor capital intelectual y cultural (Rodríguez-Ponce et al., 2018) más aún, a objeto de contar con una adecuada calidad del profesorado se requiere de una disposición para el trabajo interdisciplinario entre las Facultades y Escuelas de educación y otras unidades académicas (Medina, 2018).

En cualquier caso, más allá de la evaluación, los conocimientos y habilidades que se deben mejorar se requiere de una institucionalidad que apoye el desarrollo profesional de los docentes en servicio para perfeccionar la didáctica, el pensamiento crítico respecto a los propios conocimientos y una actualización constante en las estrategias de enseñanza de los contenidos de la disciplina (Herrada y Zapata, 2018).

La calidad de los futuros educadores de párvulos dependerá de manera decisiva de la calidad de los profesores que preparan a esos educadores en el proceso de formación inicial (Uhlenberg, 2016). Similar situación ocurre en la formación de Profesores de Enseñanza Básica, ya que la calidad de los maestros es clave en la preparación para el trabajo educativo de los futuros docentes (McLaren, 2015).

Se propone, entonces, que un factor determinante de la calidad de la formación de profesionales en general, y de las carreras de Educación Parvularia y Educación Básica en particular, lo constituye la calidad del profesorado.

\section{Recursos educativos}

Por otro lado, se ha encontrado que la infraestructura, laboratorios y equipamiento, es decir, los recursos educativos inciden en los procesos de aprendizaje. La infraestructura considera aspectos tales como: biblioteca; conectividad; laboratorios; espacios de esparcimiento; las facilidades de la sala de clases, incluyendo computadores y pizarras interactivas; y considera además el acceso a bibliografía y materiales de enseñanza (Cuesta et al., 2015). En línea con lo anterior, las facilidades y el entorno que ofrece el campus universitario influyen sobre las tasas de graduación efectiva (Horn y Lee, 2016). Según lo dicho, los procesos de formación de los educadores no pueden dejar de lado las consideraciones de equipamiento e infraestructura (Latipova et al., 2016).

En el caso específico de la Educación Parvularia, el material didáctico constituye un elemento central ya que, en el proceso de formación de niños, la comunicación mediante material educativo juega un papel fundamental para la interacción y el aprendizaje efectivo (Slot et al., 2015). Por lo tanto, en la preparación de los futuros formadores, la infraestructura, el equipamiento y el material educativo son de la mayor importancia para generar procesos educativos eficaces y eficientes (Graybill et al., 2016).

En definitiva, en su acepción más amplia, los recursos educativos tales como la infraestructura, el equipamiento y las facilidades en la sala de clases y en el entorno educativo en su conjunto, describen factores clave de la calidad de la formación de profesionales tanto para la Educación Parvularia como para la Educación Básica.

\section{Calidad de los estudiantes}

Por su parte, las condiciones de entrada de los estudiantes que ingresan al sistema de educación superior tienen también una gran relevancia en los resultados de los procesos educativos. La trayectoria previa del estudiante tales como sus calificaciones en el nivel anterior, sus resultados en exámenes estatales, y su rendimiento en la prueba selección explican el rendimiento que se logra en el sistema de educación superior (Dickinson y Adelson, 2016).

Más aún, existe un elemento clave, el que consiste en reconocer la diversidad de perfiles y atributos de la población de estudiantes universitarios lo que resulta fundamental para generar políticas y programas con una mirada más holística y no segregadora ni estigmatizadora, que permita afrontar los desafíos que estos cambios del sector de la Educación Superior provocan (Araneda et al., 2018).

Sin embargo, un acercamiento a los datos de la P.S.U. y a la medida del ranking nos muestra una realidad clara: según el Sistema Único de Admisión, en el proceso de postulación del año 2018 los alumnos de colegios particulares promediaron 626 puntos de ranking, y 611 puntos por sus notas. En tanto, los egresados de liceos tuvieron 569 puntos de ranking, y 545 puntos por las calificaciones. Nótese que en el proceso de admisión de estudiantes de la promoción 2018, un 26\% de los alumnos que obtuvo 850 puntos en el ranking, no logró 
llegar a los 500 puntos promedio en la PSU. En todo caso Bernasconi indica que el ranking "es un buen contrapeso al carácter absoluto de la PSU" (EI Mercurio, 2018).

En efecto, los resultados de la prueba de selección universitaria están determinados en gran medida por la condición socioeconómica de los alumnos (Rodríguez-Ponce, 2012). Por lo mismo, la trayectoria académica de los estudiantes y su rendimiento relativo en la enseñanza media parece ser una variable que genera mayor inclusión, siendo a la vez un indicador de excelencia académica (Gil et al., 2013). En este contexto, el puntaje en la P.S.U., ranking y el promedio de enseñanza media, junto a la ubicación relativa del estudiante, son determinantes de los resultados.

En el campo específico de la formación de profesionales de la Educación Parvularia y de la Educación Básica, la evidencia en Chile (Valencia y Taut, 2011) revela que los resultados posteriores al egreso tanto en la formación disciplinaria como en la formación pedagógica están fuertemente determinados por las condiciones de entrada a la carrera, es decir, por el puntaje promedio en la prueba de selección universitaria. En forma equivalente hasta el 2012, se ha encontrado que las universidades con mayores años de acreditación, que son precisamente las más selectivas, han tenido mejores resultados en la evaluación Inicia (Pedraja-Rejas et al., 2012). No obstante, lo anterior, aún prevalecen diferencias por nivel socio económico en: el acceso, la permanencia, los resultados académicos y la inserción laboral.

Posteriormente, a nivel nacional se ha introducido con fuerza la discusión respecto de las habilidades que se debieran potenciar en los estudiantes que ingresan a Educación Parvularia y a Educación Básica por parte de los profesores. Frente a esto, cabe mencionar que los niños que ingresaron el 2017 a Pre - kínder, egresarán de cuarto Medio el año 2030 y para esa fecha algunos trabajos van a desaparecer y todos tendrán cambios, lo que hace difícil saber las habilidades que se requerirán (Henríquez, 2018).

Sin lugar a dudas la Ley de Carrera Docente, establece un aumento en las exigencias para los estudiantes que accedan a los programas de Formación Inicial Docente. En lo concreto, la legislación establece un aumento paulatino de los puntajes mínimos para ingresar a la carrera. Su implementación partió en 2017, con un piso de 500 puntos y debería alcanzar los 550 hacia el año 2023 (La Tercera, 2018).

En consecuencia, integrar inductivamente en un modelo, la calidad del profesorado, los recursos educativos y la calidad de los estudiantes, a partir del análisis hasta aquí llevado a cabo, es pertinente y relevante.

\section{Estilos de liderazgo}

Conjuntamente, en términos teóricos, es posible incorporar al análisis anterior los estilos de liderazgo como un determinante que puede ser clave para explicar diferentes relaciones entre condiciones de entrada y procesos formativos y sus efectos sobre los resultados académicos diferenciados sistémicamente. En el estado del arte, estas dimensiones comúnmente se abordan en forma separada. A diferencia de la forma tradicional de estudiar los estilos de liderazgo, en esta propuesta teórica, se incorporan en una perspectiva de interacción con otros factores determinantes de la calidad de las carreras en análisis.

En efecto, el estilo de liderazgo constituye el patrón de conducta que moviliza a los seguidores a realizar lo que el líder les ha requerido en procura de alcanzar los objetivos institucionales (McCamish et al., 2015). Existen múltiples formas de tipificar los estilos de liderazgo, aunque la perspectiva dominante actualmente distingue entre: liderazgo transaccional, liderazgo transformacional y laissez faire (Khan et al., 2016). La evidencia internacional reconoce que el liderazgo transformacional puede incidir en las entidades educativas, principalmente en el clima, la cultura y el desempeño académico; en el nivel de logro de los estudiantes y en el compromiso de los profesores; impactando en los procesos de enseñanza y en los resultados académicos (Ling y Ling, 2016).

En el campo de la educación superior, se ha encontrado que el estilo de liderazgo influye sobre el compromiso y la satisfacción de los académicos (Trivellas y Santouridis, 2016). Por otro lado, Ersozlu y Saklan (2016) destacan la importancia del liderazgo en múltiples niveles relevando su impacto en las actitudes, comportamiento y en el aprendizaje, precisamente, en educación superior se advierten hallazgos relevantes respecto de la importancia del liderazgo para generar compromiso en los académicos (Henkel 2016).

A su vez, se ha encontrado evidencia relevante de la pertinencia de plantear una relación entre: estilos de liderazgo y calidad de las carreras universitarias, lo cual constituye un hallazgo novedoso en el campo de la educación superior en países emergentes (Pedraja-Rejas et al., 2018). Por su parte Al-Husseini y Ebeltagi (2016) muestran que los estilos de liderazgo inciden en la capacidad de innovación, incluso a nivel de procesos educativos en las universidades públicas y privadas. 
Por tanto, se sugiere que los estilos de liderazgo representan una dimensión relevante que habría de impactar sobre los procesos educativos y sobre los resultados académicos en la formación de profesionales de Educación de Párvulos y de la Educación Básica. Con todo, habría que descubrir, empíricamente en estudios futuros, en qué medida los estilos de liderazgo explican los resultados académicos para condiciones de entrada y procesos formativos específicos, constituyéndose en una variable que optimiza la diferenciación sistémica de los resultados para la Educación Parvularia y Educación Básica en Chile. En esta etapa del análisis, es posible plantear que los factores que influyen en los procesos formativos y, subsecuentemente, en los resultados de las carreras de Educación Parvularia y Profesor de Educación Básica son: 1) calidad del profesorado; 2) recursos educativos; 3) calidad de los estudiantes; y 4) estilos de liderazgo.

\section{Los procesos de formación}

En el contexto de la implementación de un programa de estudio, los procesos de formación se configuran a partir del conjunto de articulaciones, acciones y actuaciones necesarias para conseguir las metas educativas. Se trata de procesos formales que procuran generar resultados educativos, creando situaciones que estimulan el aprendizaje, ya sea en forma verbal (Tandyonomanu, 2017), mediante imágenes o múltiples métodos, en orden a que el estudiante logre aprehender y empoderarse no sólo de contenidos, sino que también de actitudes, habilidades y competencias para resolver los desafíos de una temática en particular (Biesta, 2015).

Los procesos formativos trascienden la sola interacción en clases entre profesores y estudiantes e incorporan el contexto social e institucional, siendo relevante destacar que un resultado esperado de los procesos de formación es el compromiso y la motivación para seguir aprendiendo (Boekaerts, 2016). Bajo esta mirada, Dike y Umegboro (2015) descubren que los procesos de enseñanza generan diferencias en el grado de aprendizaje de los estudiantes, por cuanto de acuerdo con los objetivos educativos y los perfiles de los alumnos existen procesos que son más apropiados que otros para lograr el aprendizaje.

Equivalentemente Wang et al. (2016) revelan que los métodos de enseñanza tienen impactos significativos en la percepción de autonomía, en la autoeficacia y en la autorregulación de los educandos, todo lo cual tiene efectos posteriores en el nivel de logro académico. La metodología de enseñanza es fundamental para entender la calidad de los procesos formativos, ya que mientras mejor se adecúe la forma de instruir a los requerimientos de aprendizaje, mayor serán los niveles de logro académico (Guilloteaux et al., 2016).

En Chile, la evaluación de la formación inicial de Educación Parvularia y de Enseñanza Básica distingue entre la formación pedagógica y la formación disciplinaria. Ésta es una diferenciación que encuentra soporte en la literatura, ya que múltiples trabajos aluden a la formación pedagógica (por ejemplo: Catalano y Catalano, (2015); Tandyonomanu, (2017)) como una dimensión independiente de la formación disciplinaria (Bharuthram y Clarence, 2015). Sin embargo, la diferenciación de estas dimensiones va más allá de vincular la formación pedagógica al cómo enseñar y la formación disciplinaria al qué enseñar, puesto que, aunque la formación pedagógica tiene su expresión más concreta en el espacio educativo y en el proceso mismo de enseñanza y aprendizaje, esto no significa que no existan componentes sustantivos de contenido en dicho proceso (Depaepe et al., 2013).

De hecho, la formación pedagógica otorga sentido y significado a la disciplina, ya que la lleva al espacio del currículum escolar. Ambas formaciones la pedagógica y la disciplinar se integran a través del diseño, implementación y evaluación de situaciones educativas en su propio contexto (Buskirk-Cohen et al., 2016). Los procesos de enseñanza son fundamentales para lograr los resultados esperados en el nivel de la educación superior. No obstante, en el caso chileno existen debilidades importantes de los procesos formativos, entre las que destacan, aquellas referidas a la deficiente preparación recibida por los estudiantes en los procesos de formación inicial, fundamentalmente en competencias para: atender la diversidad en el aula; manejar de la disciplina; y trabajar con padres y familias (Gaete et al., 2016).

En este ámbito, si se considera el documento de Bases Curriculares Educación Parvularia, generado por parte de la subsecretaría de Educación Parvularia el año 2018, es posible identificar que la familia y la comunidad educativa son, de hecho, agentes formadores de los niños en múltiples formas, constituyendo un contexto educativo en sí mismo (Subsecretaría de Educación Parvularia, 2018).

Más aún, un estudio realizado por el Observatorio Formación Docente durante el año 2017, establece que en la carrera de Pedagogía Básica la tasa de recambio de profesores en todas las regiones del país es menor al $4 \%$, lo que significa que actualmente no existe la capacidad para suplir la demanda existente en Chile (Acción Educar, 2019). 


\section{Los resultados académicos}

Una primera aproximación a los resultados académicos suele asociarse con la proporción de alumnos que egresan en comparación con quienes ingresaron al proceso educativo (Page y Scott-Clayton, 2016), sin embargo, existen otras variables significativas tales como la inserción de los titulados en el mercado del trabajo tanto en la consecución de empleo como en la renta promedio, a lo que debe añadirse la continuidad en estudios de Magíster y Doctorado.

Al mismo tiempo, en Chile, el Programa de Formación Inicial Docente del Centro de Perfeccionamiento, Experimentación e Investigaciones Pedagógicas (CPEIP) aplicaba la Evaluación Inicia como una prueba de carácter formativo y diagnóstico dirigida a estudiantes egresados de, entre otras, las carreras de Educación Parvularia y Educación Básica. Dicha evaluación constituye una evidencia valiosa para conocer el nivel de conocimientos pedagógicos y disciplinarios de los egresados y, además, ofrece una orientación adecuada para mejorar la calidad (Rivero y Hurtado, 2015).

En este sentido, resulta importante tomar en cuenta la última evaluación Inicia llevada a cabo el año 2014, la cual, por cierto, solo fue rendida por sólo el 9,18\% de la población total de egresados, más específicamente, por 377 egresados de Educación Parvularia y 876 egresados de Educación Básica. Los resultados muestran una distancia significativa respecto de niveles superiores de calidad ( $75 \%$ de logro o superior), ya que dicha categoría es alcanzada en Educación Parvularia sólo por el 13,8\% de los egresados que rindieron la prueba de conocimientos pedagógicos y sólo por el $23,6 \%$ de los participantes de la prueba de conocimientos disciplinarios; en el caso de Pedagogía en Educación Básica el nivel superior sólo lo alcanza el 25,8\% de los egresados que respondieron la prueba de conocimientos pedagógicos y sólo el $24,6 \%$ de los participantes en la prueba de conocimientos disciplinarios.

Ahora bien, desde el año 2016, como se ha planteado previamente, con la promulgación de la Ley 20.903 que establece el Sistema de Desarrollo Profesional Docente, se sabe que las diversas carreras de pedagogías solo pueden acreditarse ante la CNA y esta acreditación es un requisito obligatorio para que puedan ser impartidas (CNA, 2018).

Con estos resultados, las universidades podrán establecer mecanismos de acompañamiento y nivelación para sus estudiantes, ya que al menos un año antes del egreso, el Mineduc aplicará la Evaluación Nacional Diagnóstica para la Formación Inicial de Profesores, que está basada en estándares pedagógicos y disciplinarios. Bajo esta nueva modalidad, rendir la evaluación será un requisito de titulación para el estudiante, pero sus resultados no serán habilitantes.

Por su parte, a fines de mayo de 2018, un total de 59 carreras de Educación Parvularia y 91 carreras de Educación Básica son impartidas en instituciones de educación superior autónomas. En los registros de calidad de la Comisión Nacional de Acreditación, se puede observar que las carreras de Educación Parvularia y Educación Básica tienen niveles cercanos a la media $(63 \%$ y $62 \%$ de sus carreras acreditadas respectivamente). Dichos resultados de calidad de las carreras por tipo de pedagogía pueden apreciarse en la figura 1 .

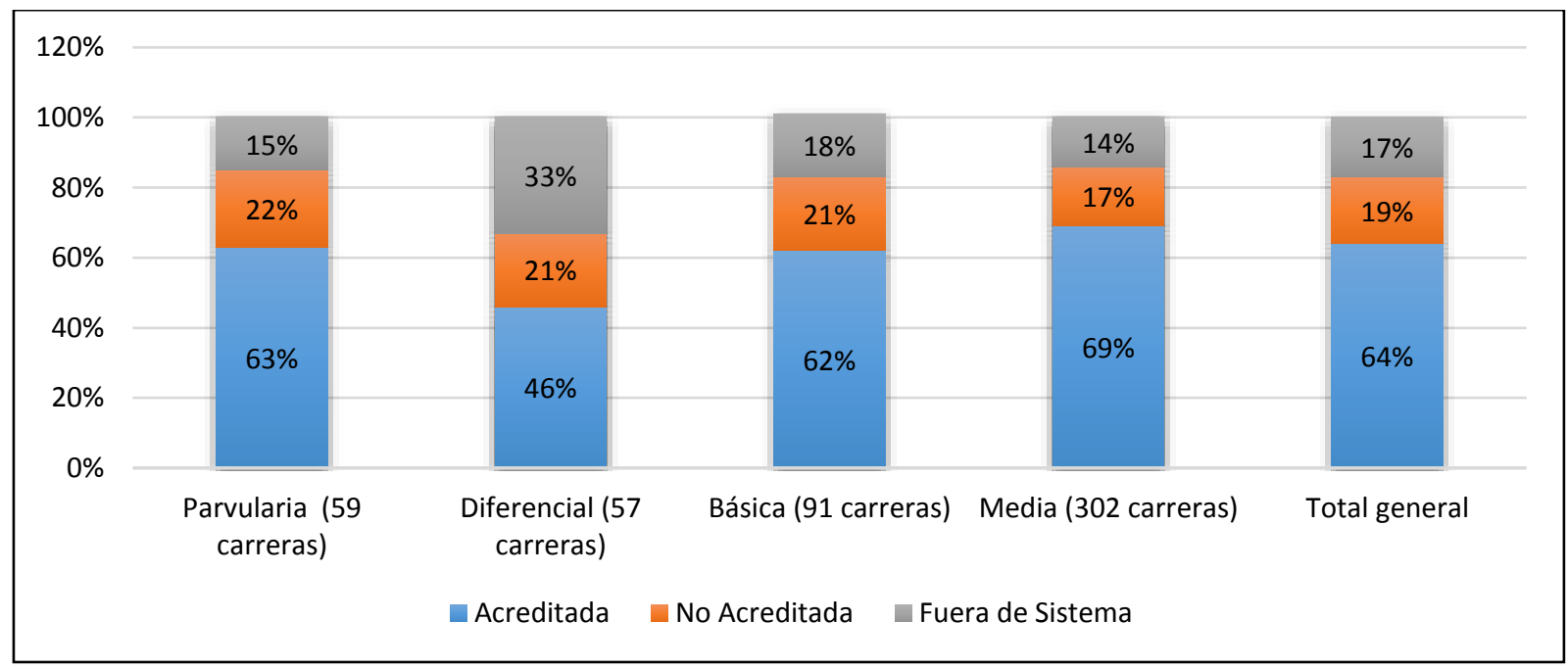

Fig.1: Acreditación de carreras del área de Educación. 
Al considerar los profesionales en ejercicio, se observan resultados equivalentes a los anteriores ya que de los 16.060 docentes de aulas que rindieron la última Evaluación Docente en 2014, sólo el 10\% alcanzó un nivel destacado, inferior incluso al 14,8\% que logró dicha categoría en 2013. En esta evaluación, un resultado particularmente revelador muestra que mientras el $88,7 \%$ de los evaluados se autocalificó como destacado, la medición específica del instrumento portafolio de trabajo en clases atribuyó dicho nivel destacado sólo al $0,1 \%$ de los evaluados (MINEDUC, 2015).

En efecto, la evaluación del 2014 es la última disponible, puesto que, la Ley 20.903 modificó la Ley de Aseguramiento de la Calidad de la Educación (Ley $N^{\circ}$ 20.129) y tal como se planteó en la introducción del presente artículo, para obtener la acreditación de carreras y programas, las universidades deben participar obligatoriamente de dos evaluaciones diagnósticas sobre formación inicial en pedagogía; una al inicio de la carrera y otra al menos un año antes del egreso (CPEIP, 2018a).

Así, el proceso de aplicación de la Evaluación Nacional Diagnóstica 2017 consideró las siguientes pruebas como obligatorias a ser rendidas por los estudiantes: 1) Prueba de Conocimientos Pedagógicos para Educación Parvularia y para Pedagogía en Educación Básica, 2) Prueba de Conocimientos Disciplinarios y Didácticos para Educación Parvularia y para Pedagogía en Educación Básica. y 3) Prueba de Reflexión Pedagógica también para ambas carreras (CPEIP, 2018b).

Como lo establece la ley, rendir esta evaluación es un requisito para la titulación. Los resultados generales y por institución son entregados a la Comisión Nacional de Acreditación, con el fin de que las universidades establezcan, de ser necesario, planes de mejora los cuales serían considerados para el diseño de acciones formativas por parte del CPEIP.

Tal como lo representa el informe nacional de Evaluación Diagnóstica de la Formación Inicial Docente 2017, los resultados que obtienen las instituciones formadoras de profesores deben ser observados en consideración a las características de cada una de estas, como, por ejemplo: tipo de institución, años de acreditación institucional, trayectoria institucional, entre otros criterios.

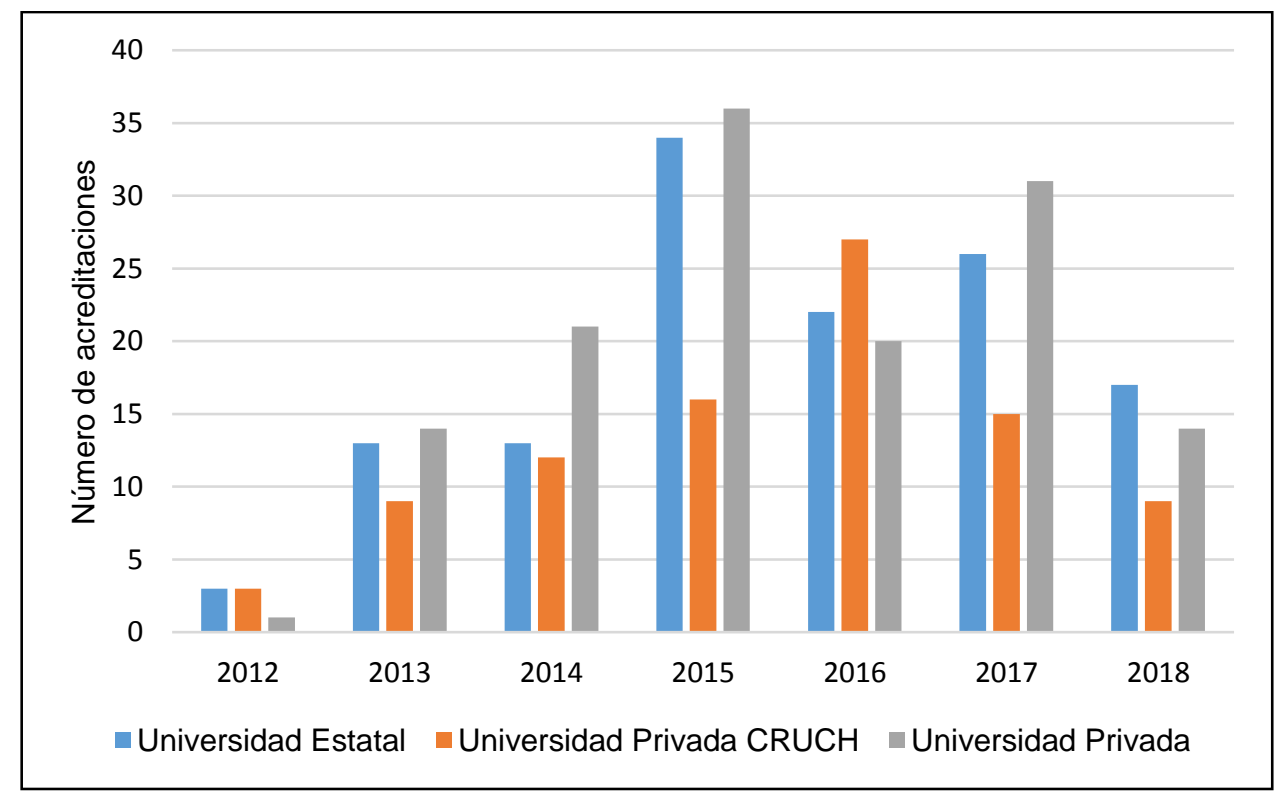

Fig. 2: Número de carreras acreditadas por tipo de institución (Datos tomados de Acción Educar, Impacto de la nueva ley de carrera docente sobre las carreras de educación superior ligadas al área de educación, 2019).

Como se puede apreciar en la figura 2, las universidades privadas desde el año 2013 al 2015 contaban con el mayor número de acreditaciones. Recién el año 2017 estas retornan liderando el número de acreditaciones.

Por otro lado, dentro de los cambios implementados producto de la puesta en marcha de la Ley de Carrera Docente, se tiene que la acreditación pasó a ser un requisito obligatorio para poder impartir las carreras de Pedagogía y que sólo las universidades podrían dar títulos de profesor (Acción educar, 2019). Este aspecto posee una implicancia esencial para el caso de las carreras de Educación Parvularia y la de Pedagogía en Educación Básica, toda vez que, a partir de la citada ley, se observa una disminución en el promedio de años de acreditación obtenido por las carreras, más pronunciado en las universidades privadas fuera del Consejo de Rectores y en las universidades estatales. Además, llama la atención que Cerca de 14.000 estudiantes de 
Pedagogía asisten a una institución que no se encuentra acreditada. En total 183 carreras de Pedagogía no se encuentran acreditadas y arriesgan cierre (de un total de 509).

En definitiva, con estas grandes modificaciones del punto de vista legal se tiene un gran desafío para la obtención de resultados académicos. De acuerdo a lo planteado por Beale (2018) se cuenta con un periodo de 3 años para obtener la acreditación institucional y de la carrera, el cual se cumple en el mes de abril 2019.

\section{CONSTRUCCIÓN DE UN MODELO INTEGRADOR}

La discusión bibliográfica realizada a partir de bases de datos, artículos del gobierno de Chile y revistas de corriente principal, permite integrar cada uno de los elementos para proponer un conjunto de hipótesis, las que, a su vez, permiten construir mediante inducción analítica un modelo integrador respecto de las relaciones entre determinantes, procesos y resultados de la formación inicial de profesores de enseñanza básica y Educación Parvularia.

Dicho de modo claro, la hipótesis central que se deriva de la investigación bibliográfica es que los resultados académicos de las carreras de Educación Parvularia y Pedagogía en Educación Básica estarían determinados tanto por las condiciones de entrada como por los procesos académicos. De modo específico y en concordancia con la revisión teórica precedente, se proponen como resultado del análisis conceptual llevado a cabo algunas hipótesis y luego la figura 3 las integra.

Hipótesis 1: La calidad del profesorado (estudios formales, experiencia, conocimientos, habilidades y actitudes) tendría un impacto sobre los procesos formativos (pedagógicos y disciplinarios) y sobre los resultados académicos (tasa de titulación, inserción laboral o continuidad de estudios, evaluación Inicia, acreditación) en las carreras de Educación Parvularia y Pedagogía en Educación Básica.

Hipótesis 2: Los recursos educativos (infraestructura, equipamiento, facilidades, entorno educativo, material educativo tendrían, un impacto sobre los procesos formativos (pedagógicos y disciplinarios) y sobre los resultados académicos (tasa de titulación, inserción laboral o continuidad de estudios, evaluación Inicia, acreditación) en las carreras de Educación Parvularia y Pedagogía en Educación Básica.

Hipótesis 3: La calidad de los estudiantes (rendimiento en educación media, ranking, pruebas de selección) tendría un impacto sobre los procesos formativos (pedagógicos y disciplinarios) y sobre los resultados académicos (tasa de titulación, inserción laboral o continuidad de estudios, evaluación egresados, acreditación) en las carreras de Educación Parvularia y Pedagogía en Educación Básica.

Hipótesis 4: Los estilos de liderazgo de los decanos o directores de Facultades (transformacional, transaccional) tendrían un impacto sobre los procesos formativos (pedagógicos y disciplinarios) y sobre los resultados académicos (tasa de titulación, inserción laboral o continuidad de estudios, evaluación egresados, acreditación en las carreras) de Educación Parvularia y Pedagogía en Educación Básica.

Hipótesis 5: Los procesos formativos (pedagógicos, disciplinarios) tendrían un impacto sobre los resultados académicos (tasa de titulación, inserción laboral o continuidad de estudios, evaluación egresados, acreditación) en las carreras de Educación Parvularia y Pedagogía en Educación Básica.

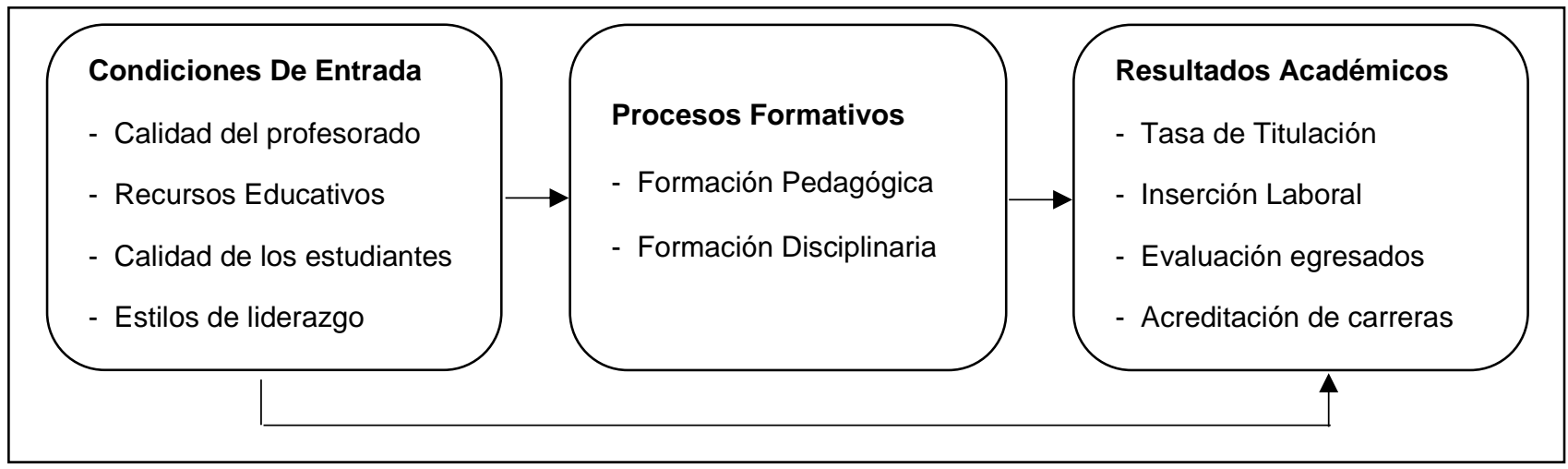

Fig.3: Modelo Integrador.

Cabe destacar que el modelo generado mediante inducción analítica, es de naturaleza integradora y sistémica, ya que plantea un enfoque integrador de variables que es novedoso y que considera, a su vez, un análisis conjunto de todas ellas, así como de sus potenciales interacciones. 


\section{DISCUSIÓN FINAL}

Las capacidades existentes en Chile para insertarse efectivamente en la sociedad del conocimiento se encuentran con una limitación crucial dada por el bajo nivel de eficacia funcional de la población nacional, esto implica que el sistema educativo no proporciona los niveles mínimos de suficiencia para enfrentar las exigencias de la sociedad del conocimiento. La Educación Parvularia y la Educación Básica constituyen la piedra angular de la preparación intelectual de las personas, así como de su posicionamiento y potencial contribución a la sociedad, sin embargo, la evidencia revisada para el caso de Chile da cuenta que las carreras, los egresados recientes y los titulados en ejercicio, en general, poseen niveles de calidad inferiores a los requeridos por una sociedad que, a su vez, exige mayor competitividad, una preparación intelectual más elevada y niveles superiores de eficacia social y funcional.

El aspecto central en términos del contexto chileno es la aprobación de la ley 20.903 que crea el sistema de Desarrollo Profesional Docente, esta, entre otros temas relevantes, establece la acreditación obligatoria para todas las carreras de pedagogía, además de instaurar dos evaluaciones a los estudiantes una al inicio de su carrera y otra un año antes del egreso de los futuros profesionales, por lo que se considera una variable relevante en el modelo. Asimismo, se determina que la calidad del profesorado, en un contexto de masificación de la educación superior, también se constituye en un aspecto relevante para aportar al resultado académico. Por tanto, para abordar el problema del bajo nivel de calidad en Educadores de Párvulos y docentes de Educación Básica se requiere de una reestructuración de las políticas educativas que implique el desarrollo de las competencias y habilidades necesarias para mejorar los procesos formativos de los futuros docentes (Salazar-Gómez y Tobón, 2018), el cual considere la definición de los criterios mínimos para el ingreso a las carreras analizadas; la especificación de los estándares de acreditación y la identificación de los requerimientos estratégicos para los estilos de liderazgo.

\section{CONCLUSIONES}

De acuerdo al análisis conceptual llevado a cabo, se puede concluir que actualmente Chile se encuentra en deuda con la formación de capital humano idoneo debido principalmente a la deficiente formación del profesorado por lo que el país debería enfocar sus esfuerzos en efectuar una formación pedagógica y disciplinaria de calidad en las carreras de Educación Parvularia y de Pedagogía en Educación Básica, a través de la cual se pueda lograr el máximo potencial de los estudiantes para que desarrollen desde pequeños habilidades y competencias requeridas en la sociedad del conocimiento. En este sentido, esta investigación propone, a partir de la generación de un modelo integrador, que los resultados académicos, tales como las tasas de titulación, la inserción y evaluación de estas carreras estarían determinados tanto por las condiciones de entrada como por los procesos académicos. Así, se postula que la articulación de las relaciones entre estas variables contribuiría a alcanzar resultados académicos diferenciados entre sí, sistémicamente.

\section{AGRADECIMIENTOS}

Este trabajo es la resultante de la ejecución del Proyecto FONDECYT 1180484 de la Comisión Nacional de Investigación Científica y Tecnológica (CONICYT).

\section{REFERENCIAS}

Acción Educar, Impacto de la Nueva Ley de Carrera Docente sobre las Carreras de Educación Superior ligadas al Área de Educación, Análisis, Chile, enero (2019)

Al-Husseini, S. y I. Elbeltagi, Transformational Leadership and Innovation: A Comparison Study between Iraq's Public and Private Higher Education, doi: 10.1080/03075079.2014.927848, Studies in Higher Education, 41(1), 159-181 (2016)

Araneda-Guirriman, C., J. Gairín-Sallán, L. Pedraja-Rejas y E. Rodríguez-Ponce, Percepciones sobre el Perfil del Estudiante Universitario en el Contexto de la Educación Superior de Masas, Interciencia, ISSN: 0378-1844, 43(12), 864870 (2018)

Ávalos, B., Initial Teachers' Education in Chile: Tensions between Policies of Support and Control, doi: 10.4067/S071807052014000200002, Estudios pedagógicos (Valdivia), 40 (Especial), 11-28 (2014)

Barber M. y M. Mourshed, How the World's Best-Performing Schools Come out on top, McKinsey \& Company, New York, USA (2007)

Beale P., Seminario Internacional, Formación Inicial Docente y Aseguramiento de la Calidad. Impacto y Desafíos de la Política Pública en el Marco de una nueva Ley de Carrera Docente en Chile, Santiago, Chile, 16 de noviembre (2018)

Bharuthram, S. y S. Clarence, Teaching Academic Reading as a Disciplinary Knowledge Practice in Higher Education, South African Journal of Higher Education, ISSN: 1011-3487, 29(2), 42-55 (2015) 
Biesta, G., What is Education For? On Good Education, Teacher Judgement, and Educational Professionalism, doi: 10.1111/ejed.12109, European Journal of Education, 50(1), 75-87 (2015)

Boekaerts, M., Engagement as an Inherent Aspect of the Learning Process, doi: 10.1016/j.learninstruc.2016.02.001, Learning and Instruction, 43, 76-83 (2016)

Buskirk-Cohen, A. A., T. A. Duncan y M. Levicoff, Using Generational Theory to Rethink Teaching in Higher Education, doi: 10.1080/13562517.2015.1110787, Teaching in Higher Education, 21(1), 25-36 (2016)

Catalano, H. y C. Catalano, The Contribution of Pedagogical Teaching Practice Activities on the Development of Communicative Competence of the Students Future Teachers for Preschool and Primary School-Ascertaining Study, doi: 10.1016/j.sbspro.2015.11.265, Procedia-Social and Behavioral Sciences, 209, 109-115 (2015)

Center on the Developing Child at Harvard University, A science-based framework for early childhood policy: Using evidence to improve outcomes in learning, behavior, and health for vulnerable children (2007)

Centro Microdatos de la Universidad de Chile, Segundo Estudio de Competencias Básicas de la Población Adulta 2013 y comparación Chile 1998-2013, Corporación de Capacitación de la Construcción y Centro Microdatos, Santiago, Chile (2014)

Clark, S. K., S. R. Helfrich y L. Hatch, Examining Preservice Teacher Content and Pedagogical Content Knowledge Needed to Teach Reading in Elementary School, doi:10.1111/1467-9817.12057, Journal of Research in Reading, 40(3), 219-232 (2016)

CNA- Comisión Nacional de Acreditación, Carreras de Pedagogía: Análisis de Fortalezas y Debilidades en el Escenario Actual. Serie Estudios sobre Acreditación, ISSN: 0719-9678, Santiago, Chile (2018)

Council for Early Childhood Development, Early Childhood Development: Southeast Regional Intersectoral Committee (2011)

CPEIP- Centro de Perfeccionamiento, Experimentación e Investigaciones Pedagógicas, Estudiantes de Pedagogía, Santiago, Chile (2018a)

CPEIP- Centro de Perfeccionamiento, Experimentación e Investigaciones Pedagógicas, Resultados Evaluación Nacional Diagnóstica de la Formación Inicial Docente 2017, Santiago, Chile, junio (2018b)

Cuesta, A., P. Glewwe y B. Krause, School Infrastructure and Educational Outcomes: A Literature Review, with Special Reference to Latin America, Economia, 17(1), 95-130 (2015)

Depaepe, F., L. Verschaffel y G. Kelchtermans, Pedagogical Content Knowledge: A Systematic Review of the way in which the Concept has Pervaded Mathematics Educational Research, doi: 10.1016/j.tate.2013.03.001, Teaching and Teacher Education, 34, 12-25 (2013)

Desimone, L. M. y M. S. Garet, Best Practices in Teachers' Professional Development in the United States, ISSN: 2171 2085, Psychology, Society, \& Education, 7(3), 252-263 (2016)

Dickinson, E. R. y J. L. Adelson, Choosing Among Multiple Achievement Measures Applying Multitrait-Multimethod Confirmatory Factor Analysis to State Assessment, ACT, and Student GPA Data, ISSN: 1932-202X, Journal of Advanced Academics, 27(1), 4-22 (2016)

Dike, J. W. y K. Umegboro, Teaching Variables and Students Performance on the Concept of Saponification, British Journal of Education, ISSN: 2054-6351, 3(8), 40-46 (2015)

El Mercurio, Diferencias entre Puntajes PSU y Ranking abren debate sobre Posible Inflación de Notas en los Colegios, Economía y Negocios Online, 28 de diciembre (2018)

Ersozlu, A. y E. Saklan, Instructional Leadership in Higher Education: How Does It Work?, British Journal of Education, ISSN: 2055-0219, 4(5), 1-15 (2016)

Gaete, A., V. Gómez y M. Bascopé, ¿Qué le piden los Profesores a la Formación Inicial en Chile?, Temas de la Agenda Pública, ISSN: 0718-9745, 11(86), 15 (2016)

Garrity, S. M., S. L. Longstreth, N. S. Salcedo-Potter y A. Staub, Using the Teaching and Guidance Policy Essentials Checklist to Build and Support Effective Early Childhood Systems, doi:10.1007/s10643-015-0713-6, Early Childhood Education Journal, 44(3), 209-216 (2016)

Gil, F., R. Paredes y I. Sánchez, El Ranking de las Notas: Inclusión con Excelencia, Temas de la Agenda Pública, ISSN: 0718-9745, 8(60), 3-19 (2013)

Graybill, E., S. Self-Brown y otros 4 autores, Addressing Disparities in Parent Education: Examining the Effects of Learn the Signs/Act Early Parent Education Materials on Parent Outcomes, doi:10.1007/s10643-014-0680-3, Early Childhood Education Journal, 44(1), 31-38 (2016)

Guilloteaux, M. J., Student Engagement during EFL High School Lessons in Korea: An Experience-Sampling Study, doi:10.15334/FLE.2016.23.1.21, Foreign Languages Education, 23(1), 21-46 (2016) 
Henkel, T., The Relationship between Transformational Leadership Styles and University Adjunct Faculty Work Engagement. Embry-Riddle Aeronautical University (2016)

Henríquez, C., Seminario Internacional, Una Mirada Amplia de Calidad: La Importancia de las Habilidades Socioemocionales, Chile, abril (2018)

Herrada, M. y A. Zapata, ¿Qué nos dice el Portafolio acerca de las mejoras en el Desempeño Docente?, Midevidencias, ISSN: 0719-8493, 1, 1-7 (2018)

Horn, A. S. y G. Lee, The Reliability and Validity ff Using Regression Residuals to Measure Institutional Effectiveness in Promoting Degree Completion, doi: 10.1007/s11162-015-9394-7, Research in Higher Education, 57(4), 469-496 (2016)

Khan, Z., A., A. Nawaz y I. Khan, Leadership Theories and Styles: A Literature Review, Journal of Resources Development and Management, ISSN: 2422-8397, 16, 1-7 (2016)

La Tercera, Nuevas exigencias complican futuro de educadoras de párvulo, 28 de septiembre (2018)

Latipova, L. A., I. E. Krapotkina y E. L. Koudrjavtseva, Research Project "Subject Developing Environment of Preschool Education" for Russian Preschool Bilinguals, doi: 10.12973/ijese.2016.364a, International Journal of Environmental \& Science Education, 11(7), 1589-1596 (2016)

Ling, S. y M. Ling, The Influence of Transformational Leadership on Teacher Commitment Towards Organization, Teaching Profession, and Student Learning in Secondary Schools in Miri, Sarawak, Malaysia, Educare, ISSN: 1979-7877, 4(2), 155$178(2016)$

Magnuson, K. y G. J. Duncan, Can Early Childhood Interventions Decrease Inequality of Economic Opportunity? doi: 10.7758/rsf.2016.2.2.05, Journal of the Social Sciences, 2(2), 123-141 (2016)

McCamish, C., H. Reynolds, B. Algozzine y D. Cusumano, An Investigation of Characteristics, Practices, and Leadership Styles of PBIS Coaches, Journal of Applied Educational and Policy Research, ISSN: 1930-9325, 1(1), 15-34 (2015)

McLaren, P., Life in schools: An introduction to critical pedagogy in the foundations of education, $6^{\text {a }}$ Ed., Routledge, New York, USA (2015)

Medina, L., A casi dos años de la promulgación de la ley 20.903: Avances, tensiones y desafíos del sistema de desarrollo profesional docente; in Ideas en educación II: Definiciones en tiempos de cambio by I. Sánchez, pp 257-290, Ediciones UC, Santiago, Chile (2018)

MINEDUC- Ministerio de Educación, Resultados Evaluación INICIA 2014, Santiago, Chile (2015)

Observatorio Formación Inicial Docente, Informe OFD N7: Localizando la Caída de la Matrícula en las Carreras de Educación y su Vínculo con la Distribución de "Beneficios de Financiamiento Universitario", enero (2019)

OCDE- Organización para la Cooperación y el Desarrollo Económico, OECD Economic Surveys: Chile 2018, doi: 10.1787/eco_surveys-chl-2018-en (2018)

Page, L. y J. Scott-Clayton, Improving college access in the United States: Barriers and policy responses, doi: 10.1016/j.econedurev.2016.02.009, Economics of Education Review, 51, 4-22 (2016)

Parker, A., A. Bush y D. Yendol-Hoppey, Understanding Teacher Candidates' Engagement with Inquiry-Based Professional Development: A Continuum of Responses and Needs, doi: 10.1080/1547688X.2015.1027978, The New Educator, 12(3), 1-22 (2016)

Pedraja-Rejas, L., C. Araneda, E. Rodríguez y J. Rodríguez, Calidad en la Formación Inicial Docente: Evidencia Empírica en las Universidades Chilenas, doi: 10.4067/S0718-50062012000400003, Formación Universitaria, 5(4), 15-26 (2012)

Pedraja-Rejas, L., R. Vega-Massó y J. Riquelme-Castañeda, La Importancia de los Estilos de Liderazgo en la Calidad de las Unidades Académicas Universitarias, Revista Opción, ISSN: 1012-1587, 34(86), 131-151 (2018)

Popescu, G. H., M. Comănescu y O. Sabie, The Role of Human Capital in the Knowledge-Networked Economy, doi: 10.22381/pihrm4120168, Psychosociological Issues in Human Resource Management, 4(1), 168-174 (2016)

Rivero, R. y C. Hurtado, Indicadores de Resultados de los Programas de Pedagogía Básica: Evidencia para Mejorar el Aseguramiento de la Calidad de los Docentes en Chile, doi: 10.31619/caledu.n43.43, Calidad en la Educación, (43), 17$55(2015)$

Rodríguez B., P. Mahias y otros 4 autores, La mirada de los Profesores: Debilidades que reconocen en su Práctica y cómo proponen Superarlas, Midevidencias, ISSN: 0719-8493, 5, 1-6 (2016)

Rodríguez-Ponce, E., L. Pedraja-Rejas, J. Rodríguez-Ponce y F. Ganga-Contreras, Relation between Selectivity, Teacher Provision and Graduation Rate in Higher Education: An Empirical Study of the State of California, Interciencia, 43(4), 249$255(2018)$

Rodríguez-Ponce, E., La Educación Superior en Chile y el Rol del Mercado: ¿Culpable o Inocente?, doi: 10.4067/S071833052012000100013, Revista Chilena de Ingeniería, 20(1), 126-135 (2012) 
Salazar-Gómez, E. y S. Tobón, Análisis Documental del Proceso de Formación Docente acorde con la Sociedad del Conocimiento, Espacios, ISSN: 0798-1015, 39(45), 17 (2018)

Slot, P. L., P. P. M. Leseman, J. Verhagen y H. Mulder, Associations between Structural Quality Aspects and Process Quality in Dutch Early Childhood Education and Care Settings, doi: 10.1016/j.ecresq.2015.06.001, Early Childhood Research Quarterly, 33, 64-76 (2015)

Subsecretaría de Educación Parvularia, Bases Curriculares Educación Parvularia, Santiago, Chile, febrero (2018)

Tandyonomanu, D., Verbal and Nonverbal Aspect on Teaching and Learning Process, Proceeding the 1st International Conference on Education Innovation, 1(1), 218-223 (2017)

Trivellas, P. y I. Santouridis, Job Satisfaction as a Mediator of the Relationship between Service Quality and Organisational Commitment in Higher Education, an Empirical Study of Faculty and Administration Staff, doi: 10.1080/14783363.2014.969595, Total Quality Management \& Business Excellence, 27(1-2), 169-183 (2016)

Uhlenberg, J., The Four Roles of a Master Toddler Teacher, doi:10.1080/10409289.2016.1088313, Early Education and Development, 27(2), 240-258 (2016)

Valencia, E. y S. Taut, Evidencia de Inequidad en el Acceso a los Docentes mejor Calificados en la Enseñanza Básica Chilena, Informe Técnico MIDE UC, Santiago, Chile (2011)

Vasconcellos, M. M. M. y M. R. L. de Sordi, University Teachers' Development: An (Im) Possible Task?, doi: 10.1590/180757622015.0450, Interface-Comunicação, Saúde, Educação, 20(57), 403-414 (2016)

Vlăsceanu, L., L. Grünberg y D. Pârlea, Quality Assurance and Accreditation: A Glossary of Basic Terms and Definitions, Bucharest: Unesco-Cepes (2004)

Wang, J. C. K., B. L. L. Ng, W. C. Liu y R. M. Ryan, Can being Autonomy-Supportive in Teaching Improve Students' SelfRegulation and Performance?: in Building Autonomous Learners by W. Liu, J. Wang y R. Ryan, pp 227-243, Springer, Singapore (2016) 
